\title{
Single-night stay for open radical prostatectomy
}

Gregory J. Nason; J.K. Kim; G.H. Tan; Khaled Ajib; Robert K. Nam

Division of Urology, Department of Surgery, Sunnybrook Health Sciences Centre, University of Toronto, Toronto ON, Canada

Cite as: Nason, GJ, Kim JK, Tan GH, et al. Single-night stay for open radical prostatectomy. Can Urol Assoc J 2020 August 7; Epub ahead of print. http://dx.doi.org/10.5489/cuaj.6600

Published online August 7, 2020

$* * *$

\section{Abstract}

Introduction: The aim of this study was to assess the effect of an enhanced care pathway on length of stay for open radical prostatectomy (RP) given that robotic-assisted laparoscopic prostatectomy (RALP) is not available to all patients in Canada.

Methods: A retrospective review was performed of all RPs performed. An enhanced care pathway was established for RPs in 2011. Patients were compared in the period before (20052010) and after (2011-2019) the introduction of the pathway.

Results: During the study period, 581 RPs were performed by a single surgeon with a median followup of 66.9 months (range 3-176). A total of 211 (36.3\%) RPs were performed from 20052010, while 370 (63.9\%) were performed from 2011-2019. The median age at RP was 65 years (range 44-81). Following the introduction of an enhanced care pathway, there were significant decreases in intraoperative blood loss ( $350 \mathrm{ml}$ vs. $200 \mathrm{ml}$; $\mathrm{p}=0.0001)$ and the use of surgical drains ( $90 \%$ vs. $9.5 \% ; \mathrm{p}=0.0001$ ). The median length of stay (LOS) over the whole study period was one day (range 1-7), which significantly decreased with the enhanced care pathway (three days vs. one day; $\mathrm{p}=0.0001)$. Since introducing the enhanced care pathway in 2011, $344(93 \%)$ patients were discharged day 1 following surgery. There were no differences in post-discharge presentations to the emergency department $(5.7 \%$ vs. $9 \% ; \mathrm{p}=0.15)$ or 30 -day readmission rates (3.8\% vs. $3.8 \% ; \mathrm{p}=1.00)$.

Conclusions: A single-night stay for open RP is safe and achievable for most patients. A dedicated, multifaceted pathway is required to attain targets for a safe and timely discharge. 


\section{Introduction}

Prostate cancer is the most common form of non-cutaneous cancer among Canadian men. It is estimated 23,300 new cases will be diagnosed in 2020, representing $20 \%$ of all cancers diagnosed in $\operatorname{men}^{1}$.

Radical prostatectomy (RP) remains one of the main treatment strategies for all stages of non-metastatic prostate cancer ${ }^{2,3}$. For low risk disease- active surveillance (AS) remains the preferred option however RP is an option for patients who decline AS. For intermediate risk disease, RP is a curative option for patients with $>10$ years life expectancy. While for high risk disease, RP is an option as part of a potential multimodality approach. RP can be performed with open, laparoscopic or robotic techniques. Although the majority of RP's in US and UK are now performed robotically ${ }^{4-6}$ - there is no level 1 evidence to support any oncological or functional benefit $^{7,8}$. There are however some clinically meaningful benefits such as shorter length of stay (LOS), decreased blood loss, transfusion rates and analgesic requirements ${ }^{8-10}$. LOS is variable depending on surgeon, hospital and country however LOS reported with RALP is approximately 1 day, although some expert centers are now reporting same day surgery whereas open RP is upwards of 2 days ${ }^{7,11-13}$.

Currently, robotic-assisted laparoscopic prostatectomy (RALP) is only available through philanthropic donor programs. A health technology assessment was performed in 2017 and opted not to provide public funding for RALP given 'the costs of using the robotic system were relatively large while the health benefits were deemed relatively small' ${ }^{11}$. RALP is however funded in other provinces such as Alberta.

The aim of this study was to assess the effect of an enhanced care pathway on length of stay for open radical prostatectomy given that RALP is not available to all patients in Canada.

\section{Methods}

A retrospective review was performed of the institutional case log to identify all radical prostatectomies performed by a single surgeon (RN) from January 2005 to September 2019. Patient demographics, operative details and pathological characteristics were collated from each patient's electronic chart, detailed in Table 1. Follow-up was determined from the date of surgery until date of last clinic follow-up or death.

An enhanced care pathway was established for radical prostatectomies in 2011. The pathway promoted a single night LOS in a short stay unit. The extra-peritoneal operative technique remained consistent throughout the study period via a lower midline incision. Pelvic lymph node dissections were performed based upon nomogram calculated risk of lymphovascular invasion. Closed suction drains were used sparingly at the surgeon's discretion. Patients were fasted from midnight and received prophylactic low molecular weight heparin and antibiotics at induction of general anesthesia. Local anesthetic (20mls of $0.25 \%$ bupivacaine) was infiltrated to the wound at the end of the procedure. Patients were ambulated the same day and diet was advanced as tolerated. Patients were discharged with a Foley catheter for 14 days. 
Analgesia requirements were managed by the urology service- post-operatively patients routinely received a combination of acetaminophen, ketorolac and hydromorphone (as required). Patients were discharged with a prescription for acetaminophen and 10 tablets of hydromorphone $1 \mathrm{mg}$ as required as well as oxybutynin for bladder spasms. To meet discharge criteria- patients needed to be mobile, tolerating diet and comfortable on oral analgesia. Patients were discharged home and not to a step down facility.

Patients were compared in the period before (2005-2010) and after (2011-2019) the introduction of the enhanced care pathway. Categorical variables such Gleason score, recurrence and readmission rates were summarized with counts and percentages. Continuous variables such as age at diagnosis, PSA, blood loss, LOS and follow-up will be summarized with median and range. The level of significance was set at $\mathrm{p}=0.05$. Statistical analyses were performed using version 9.4 of the SAS system for Windows (2002-2012 SAS Institute, Inc., Cary, NC). The study was approved by institutional review board.

\section{Results}

During the study period, 581 RPs were performed by a single surgeon with a median follow up of 66.9months (Range 3-176). 211 (36.3\%) RPs were performed from 2005 to 2010. At the beginning of 2011, an enhanced care pathway was introduced and 370 (63.9\%) RPs were performed from then until September 2019. The patient demographics and histopathological characteristics are detailed in Table 1.

The median age at RP was 65 years (Range 44-81). The median PSA at diagnosis was 6.6ng/dL (Range $0.25-273)$. There was no difference in PSA between the two study periods (6.7 vs 6.6, $\mathrm{p}=$ $0.31)$.

Disease was organ confined (pT2) in 335 patients (57.7\%), with more organ confined disease in the earlier time period ( $64 \%$ vs $54.1 \%, \mathrm{p}=0.02$ ). Negative surgical margins were achieved in 437 (75.2\%) patients, which remained consistent throughout the study (76.8\% vs $74.3 \%, \mathrm{p}=0.55)$. An undetectable PSA was detected in $493(84.9 \%)$ of patients, (82\% vs $86.5 \%$, $\mathrm{p}=0.15)$. A biochemical recurrence occurred in $157(27 \%)$ patients $(30.8 \%$ vs $24.9 \%, \mathrm{p}=0.14)$ while $121(20.8 \%)$ patients received adjuvant/salvage radiation ( $24.6 \%$ vs $18.6 \%, \mathrm{p}=0.09)$. Following the introduction of an enhanced care pathway- there were significant decreases in intra-operative blood loss ( $350 \mathrm{mls}$ vs $200 \mathrm{mls}, \mathrm{p}=0.0001$ ) and the use of surgical drains ( $90 \%$ vs $9.5 \%, \mathrm{p}=0.0001$ ), Table 2 . The median LOS over the whole study period was 1 day (Range 1-7) which significantly decreased with the enhanced care pathway (3days vs 1 day, $\mathrm{p}=0.0001$ ). Since introducing the enhanced care pathway in 2011, 344 (93\%) patients were discharged day 1 following surgery.

There were no differences in post discharge presentations to the Emergency Department $(5.7 \%$ vs $9 \%, \mathrm{p}=0.15)$ or 30 day readmission rates $(3.8 \%$ vs $3.8 \%, \mathrm{p}=1.00)$. There was however a significant reduction in 90day readmission rates $(11.4 \%$ vs $5.1 \%, \mathrm{p}=0.008)$. 


\section{Discussion}

RALP is the foremost approach for RP in many jurisdictions. Currently in Ontario, it is not publically funded. Herein, we performed a retrospective analysis of a high volume open surgeon's experience with RP in a unit with an enhanced care pathway. Our results demonstrate that open RP can be performed with a single night LOS in the majority of patients- a LOS similar to most RALP series.

The enhanced care pathway was associated with a 2 day decrease in LOS. An initial concern was that by discharging patients home early that there would reflexively be an increase in presentations to the Emergency Department and a higher readmission rate- neither was evident in our study. In fact, the 90 day readmission rate decreased. Some centres in North America discharge patients to a step-down facility which obviously aids an early discharge- that is not the practice at our institution- patients are discharged home without any routine home nursing follow-up. A 2 day decrease in LOS results in substantial health economic savings as well as increasing bed availability in our overloaded Canadian health service.

Cost is a significant factor with regards to the establishment of any new program- more importantly in a publically funded system. Some of the advocates of RALP offset the capital and maintenance costs associated with the robotic surgical system against the presumed decreased $\operatorname{LOS}^{14}$. A Cochrane review also demonstrated open RP was associated with a longer length of stay (mean difference 1.72 days) ${ }^{15}$. In an analysis of nearly 70,000 RPs in the United States between 2010 and 2015, RALP was associated with a higher peri-operative cost compared to open RP $\left(\sim 3000 /\right.$ case) however a shorter LOS (mean difference $\sim 1$ day) ${ }^{16}$. Similar higher costs were apparent in a 10 year study of 630,000 RPs across 49 hospitals in the United States, RALP was $\sim \$ 4500$ more expensive than open RP primarily due to operating room and disposable $\operatorname{costs}^{17}$. These costs were no longer significantly different among the highest-volume surgeons $(\geq 104$ cases/yr; $+\$ 1990, p=0.40)$ and highest-volume hospitals $(\geq 318$ cases $/ y r ;+\$ 1225, p=0.39)$. Although we did not perform a cost analysis nor have a RALP cohort for comparison- we demonstrated LOS similar to most reported RALP series.

Same day or outpatient RALP has been described by some experienced prostatectomists $^{12,18-22}$. We have yet to attempt this but do feel this may be possible in select patients (close proximity to the institution, first case of the day, fit/slim patient, social support). Abaza et al, described a series of same day discharges following RALP ${ }^{12}$. Same day discharge was managed in $49.2 \%$ of patients- the remaining patients were all discharge post operative day 1. Interestingly, $70 \%$ of the cases performed first in the day were same day discharges compared with only $2 \%$ of the third cases of the day. Of note, this is a very experienced high volume prostate service (>3000 RALPs by a single surgeon), the 500 cases described in that series were performed in an 18 month period, a similar number to our 15 year series.

Enhanced recovery after surgery (ERAS) protocols have been shown to aid early discharge, in urology these have been best described in bladder cancer for radical 
cystectomy ${ }^{22,25}$. ERAS protocols reduce morbidity and length of stay through to the implementation of multidisciplinary, peri-operative steps. Consensus has been reached for key principles regarding nutrition, anesthetic, analgesia, early mobilization ${ }^{26}$. It is difficult to identify which parameters of an ERAS protocol are most important. Although most of these protocols described pertain to robotic surgery, the principles no doubt are applicable to open surgery. The key is a multi-disciplinary pathway on achievable patient milestones (mobilization, diet, analgesia)- this combined pre-, peri- and post-operative approach focuses the patient on recovery and a planned early discharge.

This study is inherently limited by the retrospective design. Surgery was performed by a high volume open pelvic oncologist well beyond his learning curve. A referral to a unit with an established RALP program was offered to all patients at diagnosis. All patients were managed on a dedicated urology short stay unit with an emphasis on single night stay. As a result- these results may not be generalizable in a lower volume unit however we believe with an enhanced care pathway, improvements in open RP are achievable with a comparable LOS to patients undergoing RALP. No doubt RALP will become publically funded in Ontario in time however until that happens we need to strive to achieve comparable results with open RP.

\section{Conclusions}

A single night stay for open radical prostatectomy is safe and achievable for the majority of patients. A dedicated multi-faceted pathway is required to attain targets for a safe and timely discharge. 


\section{References}

1. https://www.cancer.ca/en/cancer-information/cancer-type/prostate/statistics. Accessed April 6, 2020.

2. Mottet N, Bellmunt J, Bolla M, et al. EAU-ESTRO-SIOG Guidelines on Prostate Cancer. Part 1: Screening, Diagnosis, and Local Treatment with Curative Intent. Eur Urol 2017 Apr;71(4):618-629.

3. Sanda MG, Cadeddu JA, Kirkby E, et al. Clinically Localized Prostate Cancer: AUA/ASTRO/SUO Guideline. Part II: Recommended Approaches and Details of Specific Care Options. J Urol 2018 Apr;199(4):990-997

4. Oberlin DT, Flum AS, Lai JD, et al. The effect of minimally invasive prostatectomy on practice patterns of American urologists. Urol Oncol 2016 Jun;34(6):255.e1-5.

5. Mazzone E, Mistretta FA, Knipper S, et al. Contemporary National Assessment of RobotAssisted Surgery Rates and Total Hospital Charges for Major Surgical Uro-Oncological Procedures in the United States. J Endourol 2019 Jun;33(6):438-447.

6. Khadhouri S, Miller C, Fowler S, et al. The British Association of Urological Surgeons (BAUS) radical prostatectomy audit 2014/2015 - an update on current practice and outcomes by centre and surgeon case-volume. BJU Int 2018 Jun;121(6):886-892.

7. Coughlin GD, Yaxley JW, Chambers SK, et al. Robot-assisted laparoscopic prostatectomy versus open radical retropubic prostatectomy: 24-month outcomes from a randomised controlled study. Lancet Oncol 2018 Aug;19(8):1051-1060.

8. Nyberg M, Hugosson J, Wiklund P, et al. Functional and Oncologic Outcomes Between Open and Robotic Radical Prostatectomy at 24-month Follow-up in the Swedish LAPPRO Trial. Eur Urol Oncol 2018 Oct;1(5):353-360.

9. Haese A, Knipper S, Isbarn H, et al. A comparative study of robot-assisted and open radical prostatectomy in 10790 men treated by highly trained surgeons for both procedures. BJU Int 2019 Jun;123(6):1031-1040.

10. Pompe RS, Beyer B, Haese A, et al. Postoperative complications of contemporary open and robot-assisted laparoscopic radical prostatectomy using standardised reporting systems. BJU Int 2018 Nov;122(5):801-807.

11. Health Quality Ontario. Robotic Surgical System for Radical Prostatectomy: A Health Technology Assessment. Ont Health Technol Assess Ser 2017 Jul 7;17(11):1-172. eCollection 2017.

12. Abaza R, Martinez O, Ferroni MC, et al. Same Day Discharge after Robotic Radical Prostatectomy. J Urol 2019 Nov;202(5):959-963.

13. Fracalanza S, Ficarra V, Cavalleri S, et al. Is robotically assisted laparoscopic radical prostatectomy less invasive than retropubic radical prostatectomy? Results from a prospective, unrandomized, comparative study. BJU Int 2008;101(9):1145-9.

14. Wallerstedt A, Tyritzis SI, Thorsteinsdottir T, et al. Short-term results after robot-assisted laparoscopic radical prostatectomy compared to open radical prostatectomy. Eur Urol 2015;67(4):660-70.

15. Ilic D, Evans SM, Allan CA, et al. Laparoscopic and robotic-assisted versus open radical prostatectomy for the treatment of localised prostate cancer. Cochrane Database Syst Rev 2017 Sep 12;9:CD009625. 
16. Mukherjee K, Kamal KM. Variation in prostate surgery costs and outcomes in the USA: robot-assisted versus open radical prostatectomy. J Comp Eff Res 2019 Feb;8(3):143-155.

17. Leow JJ, Chang SL, Meyer CP, et al. Robot-assisted Versus Open Radical Prostatectomy: A Contemporary Analysis of an All-payer Discharge Database. Eur Urol 2016 Nov;70(5):837-845.

18. Banapour P, Elliott P, Jabaji R, et al. Safety and feasibility of outpatient robot-assisted radical prostatectomy. J Robot Surg 2019 Apr;13(2):261-265

19. Martin AD, Nunez RN, Andrews JR, et al. Outpatient prostatectomy: too much too soon or just what the patient ordered. Urology 2010 Feb;75(2):421-4.

20. Berger AK, Chopra S, Desai MM, et al. Outpatient robotic radical prostatectomy: matched-pair comparison with inpatient surgery. J Endourol 2016 May;30 Suppl 1:S52-6.

21. Wolboldt M, Saltzman B, Tenbrink P, et al. Same-day discharge for patients undergoing robot-assisted laparoscopic radical prostatectomy is safe and feasible: results of a pilot study. J Endourol 2016 Dec;30(12):1296-1300.

22. Ploussard G, Almeras C, Beauval JB, et al. Same-day discharge surgery for robot-assisted radical prostatectomy in the era of ERAS and prehabilitation pathways: a contemporary, comparative, feasibility study. World J Urol 2020 Feb 17. doi: 10.1007/s00345-02003119-w. [Epub ahead of print]

23. Azhar RA, Bochner B, Catto J, et al. Enhanced Recovery after Urological Surgery: A Contemporary Systematic Review of Outcomes, Key Elements, and Research Needs. Eur Urol 2016 Jul;70(1):176-187.

24. Pang KH, Groves R, Venugopal S, et al. Prospective Implementation of Enhanced Recovery After Surgery Protocols to Radical Cystectomy. Eur Urol 2018 Mar;73(3):363371.

25. Lin C, Wan F, Lu Y, et al.

Enhanced recovery after surgery protocol for prostate cancer patients undergoing laparosc opic radical prostatectomy. J Int Med Res 2019 Jan;47(1):114-121.

26. Collins JW, Patel H, Adding C, et al. Enhanced Recovery After Robotassisted Radical Cystectomy: EAU Robotic Urology Section Scientific Working Group C onsensus View. Eur Urol 2016 Oct;70(4):649-660. 
Figures and Tables

\begin{tabular}{|c|c|c|c|c|}
\hline \multicolumn{5}{|c|}{ Table 1. Patient demographics and histopathological characteristics } \\
\hline & $\begin{array}{c}\text { Total } \\
(\mathbf{n}=581)\end{array}$ & $\begin{array}{c}2005-2010 \\
(n=211)\end{array}$ & $\begin{array}{c}2011-2019 \\
(n=370)\end{array}$ & $\mathbf{p}$ \\
\hline $\begin{array}{l}\text { Age at RP, years } \\
\text { (median, range) }\end{array}$ & $65(44-81)$ & $64(44-76)$ & $65(44-81)$ & 0.005 \\
\hline $\begin{array}{l}\text { PSA at diagnosis, } \\
\mathrm{ng} / \mathrm{mL} \text { (median, } \\
\text { range) }\end{array}$ & $\begin{array}{c}6.6 \\
(0.25-273)\end{array}$ & $6.7(0.25-70)$ & $6.6(1.1-273)$ & 0.31 \\
\hline \multicolumn{5}{|l|}{ Clinical stage $(\mathrm{n}, \%)$} \\
\hline $\begin{array}{l}\text { No DRE } \\
\text { performed }\end{array}$ & $3(0)$ & $0(0)$ & $3(0.8)$ & \\
\hline T1a & $1(0)$ & $1(0)$ & $0(0)$ & \\
\hline $\mathrm{T} 1 \mathrm{~b}$ & $8(0)$ & $0(0)$ & $8(2.2)$ & \\
\hline T1c & $420(72.3)$ & $152(72)$ & $268(72.4)$ & \\
\hline $\mathrm{T} 2 \mathrm{a}$ & $95(16.4)$ & $40(19)$ & $55(14.9)$ & (c) \\
\hline $\mathrm{T} 2 \mathrm{~b}$ & $22(3.8)$ & $10(4.7)$ & $12(3.2)$ & 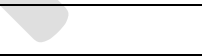 \\
\hline T2c & $8(0)$ & $1(0)$ & $7(1.9)$ & \\
\hline T3a & $20(3.4)$ & $7(5.2)$ & $13(3.5)$ & \\
\hline $\mathrm{T} 3 \mathrm{~b}$ & $2(0)$ & $0(0)$ & $2(0.5)$ & \\
\hline $\mathrm{T} 4$ & $2(0)$ & $0(0)$ & $2(0.5)$ & \\
\hline \multicolumn{5}{|c|}{ TRUS biopsy Gleason score (n, \%) } \\
\hline $\begin{array}{l}\text { No biopsy } \\
\text { performed }^{\dagger}\end{array}$ & $2(0)$ & $0(0)$ & $2(0.5)$ & \\
\hline $3+3$ & $127(21.9)$ & $75(35.5)$ & $52(14.1)$ & \\
\hline $3+4$ & $238(41)$ & $73(34.6)$ & $165(44.6)$ & \\
\hline $3+5$ & $1(0)$ & $0(0)$ & $1(0)$ & \\
\hline $4+3$ & $92(15.8)$ & $26(12.3)$ & $66(17.8)$ & \\
\hline $4+4$ & $54(9.3)$ & $17(8.1)$ & $37(10)$ & \\
\hline $4+5$ & $62(10.7)$ & $19(9)$ & $43(11.6)$ & \\
\hline $5+4$ & $2(0)$ & $0(0)$ & $2(0.5)$ & \\
\hline $5+5$ & $3(0)$ & $1(0)$ & $2(0.5)$ & \\
\hline \multicolumn{5}{|c|}{ RP Gleason score $(\mathrm{n}, \%)$} \\
\hline No score assigned & $1(0)$ & $1(0)$ & $0(0)$ & \\
\hline $3+3$ & $81(13.9)$ & $40(49.4)$ & $41(11.1)$ & \\
\hline $3+4$ & $267(46)$ & $93(44.1)$ & $174(47)$ & \\
\hline $3+5$ & $2(0)$ & $1(0)$ & $1(0)$ & \\
\hline $4+3$ & $135(23.2)$ & $45(21.3)$ & $90(24.3)$ & \\
\hline $4+4$ & $13(2.2)$ & $1(0)$ & $12(3.2)$ & \\
\hline $4+5$ & $66(11,4)$ & $24(11.4)$ & $42(11.4)$ & \\
\hline $5+4$ & $14(2.4)$ & $5(2.4)$ & $9(2.4)$ & \\
\hline $5+5$ & $2(0)$ & $1(0)$ & $1(0)$ & \\
\hline
\end{tabular}




\begin{tabular}{|c|c|c|c|c|}
\hline \multicolumn{4}{|c|}{ RP pathological stage $(n, \%)$} & \multirow[t]{2}{*}{0.02} \\
\hline $\mathrm{T} 2$ & $335(57.7)$ & $135(64)$ & $200(54.1)$ & \\
\hline $\mathrm{T} 3 \mathrm{a}$ & $145(25)$ & $50(23.7)$ & $95(25.7)$ & \\
\hline $\mathrm{T} 3 \mathrm{~b}$ & $96(16.5)$ & $24(11.4)$ & $72(19.5)$ & \\
\hline $\mathrm{T} 4$ & $5(0)$ & $2(0.9)$ & $3(0.8)$ & \\
\hline \multicolumn{4}{|c|}{ Surgical margins $(\mathrm{n}, \%)$} & 0.55 \\
\hline Negative & $437(75.2)$ & $162(76.8)$ & $275(74.3)$ & \\
\hline Positive & $144(24.8)$ & $49(23.2)$ & $95(25.7)$ & \\
\hline T2 positive & $37(11)$ & $18(13.3)$ & $19(9.5)$ & \\
\hline$\geq \mathrm{T} 3$ positive & $107(43.5)$ & $31(40.8)$ & $76(44.7)$ & \\
\hline \multicolumn{5}{|c|}{ Pathological node status (n, \%) } \\
\hline $\mathrm{Nx}$ & $281(48.4)$ & $122(57.8)$ & $159(43)$ & \\
\hline N0 & $256(44.1)$ & $82(38.9)$ & $174(47)$ & \\
\hline $\mathrm{N} 1$ & $44(7.6)$ & $7(3.3)$ & $37(10)$ & \\
\hline \multicolumn{4}{|c|}{ Postoperative PSA (n, \%) } & 0.15 \\
\hline$<0.02$ & $493(84.9)$ & $173(82)$ & $320(86.5)$ & \\
\hline$>0.02$ & $88(15.1)$ & $38(18)$ & $50(13.5)$ & \\
\hline $\begin{array}{l}\text { Biochemical } \\
\text { recurrence }(\mathrm{n}, \%)\end{array}$ & $157(27)$ & $65(30.8)$ & $92(24.9)$ & 0.14 \\
\hline $\begin{array}{l}\text { Radiation treatment } \\
(\mathrm{n}, \%)\end{array}$ & $121(20.8)$ & $52(24.6)$ & $69(18.6)$ & 0.09 \\
\hline $\begin{array}{l}\text { Followup. months } \\
\text { (median, range) }\end{array}$ & $66.9(3-176)$ & $104(3-176)$ & $38.2(3-106)$ & \\
\hline Salvage RP $(n, \%)$ & $35(6)$ & $17(8.1)$ & $18(4.9)$ & \\
\hline
\end{tabular}

*Three patients did not have a digital rectal examination, as they had previous abdomino-perineal resections and closed anus. ${ }^{\dagger}$ Two patients did not have a biopsy for the same reason above; one of those had a transperineal biopsy. The two without biopsy had PIRADS 5 lesions on MRI and a high PSA. One patient did not have a Gleason score assigned to their prostatectomy specimen due to the effects of neoadjuvant ADT and radiation treatment. ADT: androgen deprivation therapy; DRE: digital rectal examination; MRI: magnetic resonance imaging; PSA: prostate specific antigen; RP: radical prostatectomy. 


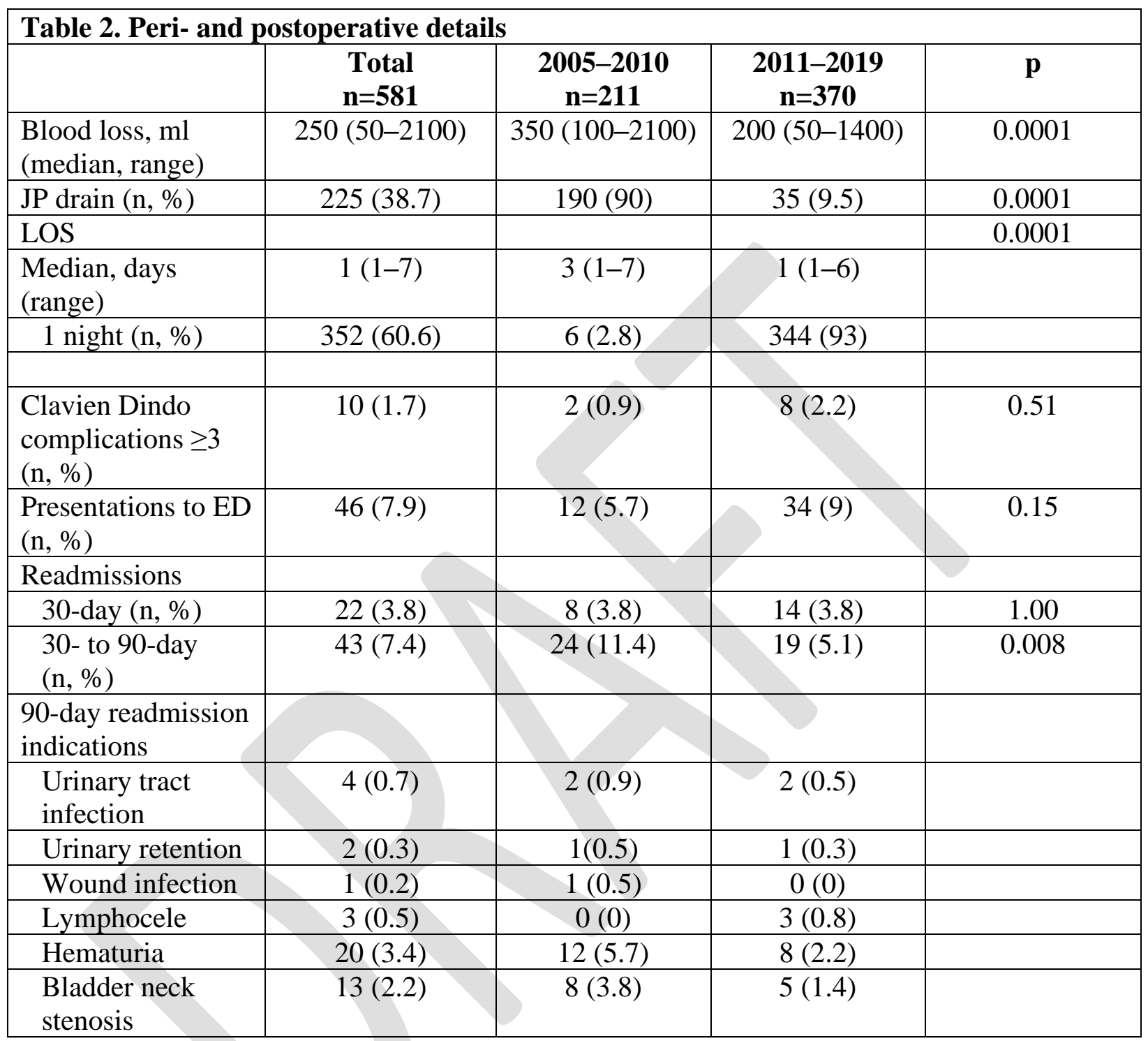

ED: emergency department; JP: Jackson Pratt; LOS: length of stay. 